\title{
DARK MATTER, AND ITS DARKNESS
}

\author{
D. V. AHLUWALIA-KHALILOVA \\ Department of Physics and Astronomy, Rutherford Building \\ University of Canterbury, Private Bag 4800 \\ Christchurch 8020, New Zealand \\ E-mail: dharamvir.ahluwalia-khalilova@canterbury.ac.nz
}

\begin{abstract}
Assuming the validity of the general relativistic description of gravitation on astrophysical and cosmological length scales, we analytically infer that the Friedmann-RobertsonWalker cosmology with Einsteinian cosmological constant, and a vanishing spatial curvature constant, unambiguously requires significant amount of dark matter. This requirement is consistent with other indications for dark matter. The same spacetime symmetries that underlie the freely falling frames of Einsteinian gravity also provide symmetries, which for the spin one half representation space, furnish a novel construct that carries extremely limited interactions with respect to the terrestrial detectors made of the standard model material. Both the 'luminous' and 'dark' matter turn out to be residents of the same representation space but they derive their respective 'luminosity' and 'darkness' from either belonging to the sector with $(C P T)^{2}=+\mathbb{1}$, or to the sector with $(C P T)^{2}=-\mathbb{1}$.

Keywords: Dark matter, Elko
\end{abstract}

\section{Introduction}

The first task that we set for ourselves in this essay is to show that the FriedmannRobertson-Walker (FRW) cosmology with $k=0$ and $\omega^{\Lambda}=-1$ requires significant amount of dark matter. This we accomplish without invoking the non-Keplerian galactic rotational curves or gravitational lensing data [1 3]. The result can be interpreted in two ways. As a complimentary requirement for the dark matter, or as a breakdown of the general relativistic description of gravitation at large astrophysical and cosmological scales 44 . Continuing the study with the former possibility, we provide a natural explanation for the darkness of dark matter. This we do by showing that the Dirac framework may suffice only for the description of the standard model fermions, and that dark matter - if we consider it fermionic, with spin one half - naturally asks for a dramatically new description where the dark matter field carries a Klein-Gordon, and not Dirac, propagator. This thesis is a natural consequence of a recent preprint [9] and two publications late last year [12, 13. The latter publications are somewhat technical, here we briefly reformulate them in a physically accessible manner. Should these results appear surprising then it suffices to note that while the abstract of Ref. 12 began as, "We report an unexpected theoretical discovery of a spin one-half matter field with mass dimension one," the abstract of Ref. [13] opened with the sentence, "We provide the first details 
of the unexpected theoretical discovery of a spin-one half matter field with mass dimension one."

For making this essay easily accessible to younger students we adopt a pedagogical style. A more knowledgeable reader may in some instances just smile, and proceed without further interruption

\section{Dark matter: A phenomenological existence proof}

Since 1997, data on supernovae 1a luminosities has built an impressive evidence for the existence of a form of energy that is accelerating the present expansion of the universe 14 19. The latest data analysis [19] favors this dark energy to be of the form postulated by Einstein in his now well-known cosmological term. The previous conflict between SN1a data and the WMAP data on anisotropies in the cosmic microwave background noted by Jassal, Bagla, and Padmanabhan 20. is now resolved [21]. Furthermore, observations on primordial cosmic microwave background and large scale structure also favor a dark energy dominated universe which is spatially flat 22,23$]$.

In the context of inhomogeneous cosmologies, Wiltshire has raised a serious objection [24, also 25] 27] to the just-stated standard interpretation of the SN 1a data and has provided an alternate interpretation which does not require dark energy [29. Working within the inflationary paradigm, he carefully distinguishes various clocks rates such as those associated with the astrophysically large gravitationally bound systems and the cosmic voids. Conceptually, the raised questions can hardly be ignored. Yet, the final verdict shall undoubtedly depend on whether such considerations turn out to be quantitatively important. Preliminary calculations are already encouraging [24, 28. So, if here we proceed with dark energy as an option it is in the spirit of an open enquiry without prejudice as to the ultimate nature of the physical reality.

In the canonical wisdom, the data thus favors a FRW cosmology where $k=$ $0, \omega^{\Lambda}=-1$ with $\rho^{\Lambda}$ as constant. However, with an exception of a few papers, it is rarely appreciated that in this cosmology the non-linearity of the Einstein field equations completely determines the proportionality constant that appears in the scale factor $a(t) \propto \sinh ^{2 / 3}\left([3 \Lambda]^{1 / 2} t / 2\right)$ [9]34. The proportionality constant depends on densities associated with matter and the Einsteinian cosmological constant $\Lambda$. The result of the analysis presented in [9, 32] is that the ratio of the corresponding densities is not arbitrary but it carries a well-determined temporal dependence. This leads to significant strengthening of the standard paradigm of the $\Lambda$ CDM cosmology, i.e the FRW cosmology defined by the set [35].

$$
\left\{k=0, w^{\Lambda}=-1, \rho=\rho_{\mathrm{m}}, p=p_{\mathrm{m}}=0, \rho^{\Lambda}=\mathrm{constant}\right\}
$$

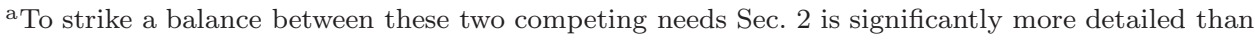
Sec. 3 This has been done because the main thesis of Sec. 3 has recently appeared in Ref. [13] in monographic detail.
} 
In particular, the stated circumstance when exploited in the context of the stellar age bound determined by the ages of the globular clusters, and the fact that fractional density associated with the standard model content $\Omega_{\mathrm{sm}} \approx 0.05$, requires existence of dark matter. These arguments are now refined and extended exploiting the recent work of [9, also 10, 11.

For the stated cosmology the Einstein field equations, when their non-linearity is fully respected [9], yield for the matter-dominated epoch

$$
\begin{aligned}
& \Omega_{\Lambda}(t)+\Omega_{\mathrm{m}}(t)=1 \\
& \Omega_{\Lambda}(t)-\zeta(t) \Omega_{\mathrm{m}}(t)=0
\end{aligned}
$$

where

$$
\zeta(t):=\sinh ^{2}\left(\frac{\sqrt{3}}{2} \frac{t}{\tau_{\Lambda}}\right)
$$

Here we introduced $\tau_{\Lambda}:=\sqrt{1 / \Lambda}$. The rest of the notation is standard, and is defined in Ref. 9] with the special attention being paid to the fact that all matter components in $\Omega_{\mathrm{m}}$ are considered non-relativistic (i.e., $p_{\mathrm{m}}=0$ ). The latter is the import of considering the 'matter dominated epoch'. Specifically, the fractional densities $\Omega_{\Lambda}$ and $\Omega_{\mathrm{m}}$ are defined as

$$
\Omega_{\Lambda}:=\frac{8 \pi G \rho^{\Lambda}}{3 H^{2}}=\frac{\Lambda}{3 H^{2}}, \quad \Omega_{\mathrm{m}}:=\frac{8 \pi G \rho_{\mathrm{m}}}{3 H^{2}}
$$

where we used $\rho^{\Lambda}:=\Lambda /(8 \pi G)$. Here, $G$ is the Newtonian gravitational constant and equals $6.7065 \times 10^{-39} \mathrm{GeV}^{-2}$. And, $H$ is the Hubble parameter. Its value for the present cosmic epoch shall be represented by $H_{0}$ below.

Equations (2) and (3) carry the following solutions

$$
\Omega_{\Lambda}=\frac{\zeta(t)}{1+\zeta(t)}, \quad \Omega_{\mathrm{m}}=\frac{1}{1+\zeta(t)}
$$

Use of the definition of $\zeta(t)$ from Eq. (4) immediately renders the above result into the form

$$
\Omega_{\Lambda}=\tanh ^{2}\left(\frac{\sqrt{3}}{2} \frac{t}{\tau_{\Lambda}}\right), \quad \Omega_{\mathrm{m}}=\operatorname{sech}^{2}\left(\frac{\sqrt{3}}{2} \frac{t}{\tau_{\Lambda}}\right)
$$

Our task for the phenomenological existence proof of dark matter now reduces to showing that $t / \tau_{\Lambda}$ for the present cosmic epoch is such that the resulting $\Omega_{\mathrm{m}}$ far exceeds that which can be accounted for by the standard model contribution of $\Omega_{\mathrm{sm}} \approx 0.05$

As such, in order to obtain these fractional densities for the present cosmic epoch we now need to constrain the cosmological constant $\Lambda$ (that yields $\tau_{\Lambda}$ ), and to obtain an expression for the age of the universe within the considered FRW cosmology. We shall first present a detailed calculation. This would be complimented by a 'back 
of the envelop' estimate to complete the phenomenological existence proof of dark matter. That would then allow us to return to the important question of its darkness.

The scale factor $a(t)$ evaluated in Ref. 9, 33, yields the present age of the universe to be 36

$$
t_{0}=\frac{2}{3 H_{0}} \frac{1}{\sqrt{\Omega_{\Lambda}\left(t_{0}\right)}} \ln \left[\frac{1+\sqrt{\Omega_{\Lambda}\left(t_{0}\right)}}{\sqrt{1-\Omega_{\Lambda}\left(t_{0}\right)}}\right]
$$

This is in agreement, e.g., with the results contained in Eq. 3.32 of Ref. 37] and in Eq. 54 of Ref. 23. In the expression for $t_{0}$, and $\Omega_{\mathrm{m}}$ evaluated at $t_{0}$, we now substitute $\Omega_{\Lambda}\left(t_{0}\right):=\Lambda /\left(3 H_{0}^{2}\right)$, and set

$$
H_{0}^{-1}=9.776 h^{-1} \text { Gyr, } \quad 0.64 \leq h \leq 0.80
$$

as obtained from the Hubble Space Telescope Key project [38]. Concurrently, we take the cosmological constant to be

$$
\Lambda=\lambda 8 \pi G 10^{-47} \mathrm{GeV}^{4}
$$

where $\lambda$ is of the order of unity (to be constrained below). This exercise gives

$$
\begin{aligned}
& t_{0}=\frac{18.55}{\sqrt{\lambda}} \ln \left[\frac{3+1.054 \sqrt{\frac{\lambda}{h^{2}}}}{\sqrt{9-1.111 \frac{\lambda}{h^{2}}}}\right] \text { Gyr } \\
& \tau_{\Lambda}=\frac{16.06}{\sqrt{\lambda}} \mathrm{Gyr} \\
& \Omega_{\mathrm{m}}\left(t_{0}\right)=\operatorname{sech}^{2}\left[\frac{1.732}{\sqrt{\lambda}} \ln \left(\frac{3+1.054 \sqrt{\frac{\lambda}{h^{2}}}}{\sqrt{9-1.111 \frac{\lambda}{h^{2}}}}\right)\right]
\end{aligned}
$$

For $\lambda \geq 2.5$ and $h=0.72$ (a suitably extended range of $\lambda$ and $h$ shall be discussed soon, below), the variations of $t_{0}$ and $\Omega_{m}\left(t_{0}\right)$ as a function of $\lambda$ are given in Figures 1 and 2. It is apparent from Fig. 1 that the demand that $t_{0}$ be greater than the stellar age bound [39, 23] of $12 \mathrm{Gyr}$, constrains the cosmological constant to $\lambda \geq 2.5$ (for $h=0.72$ ). The present cosmic epoch of $13.5 \pm 1.5$ Gyr corresponds to $2.50 \leq \lambda \leq 3.45$ (for $\mathrm{h}=0.72$ ).

Figure 2 clearly shows that for the present cosmic epoch $0.22 \leq \Omega_{\mathrm{m}} \leq 0.35$. The standard model contribution $\Omega_{\mathrm{sm}} \approx 0.05$ falls too short to account for the obtained $\Omega_{\mathrm{m}}$. This deficit is the dark matter. Its existence is here arrived at without invoking the non-Keplerian galactic rotational curves or the data on gravitational lensing [13.

To emphasize this point further it is instructive to define

$$
\alpha=\frac{\Omega_{\mathrm{m}}-\Omega_{\mathrm{sm}}}{\Omega_{\mathrm{sm}}}:=\frac{\Omega_{\mathrm{dm}}}{\Omega_{\mathrm{sm}}}
$$

In Figure 3, we exhibit its variation with $\lambda$ for $\Omega_{\mathrm{sm}}=0.05$. For the present cosmic epoch we thus find $3.3 \leq \alpha \leq 5.9$. Or, equivalently $0.17 \leq \Omega_{\mathrm{dm}} \leq 0.30$. That is, 


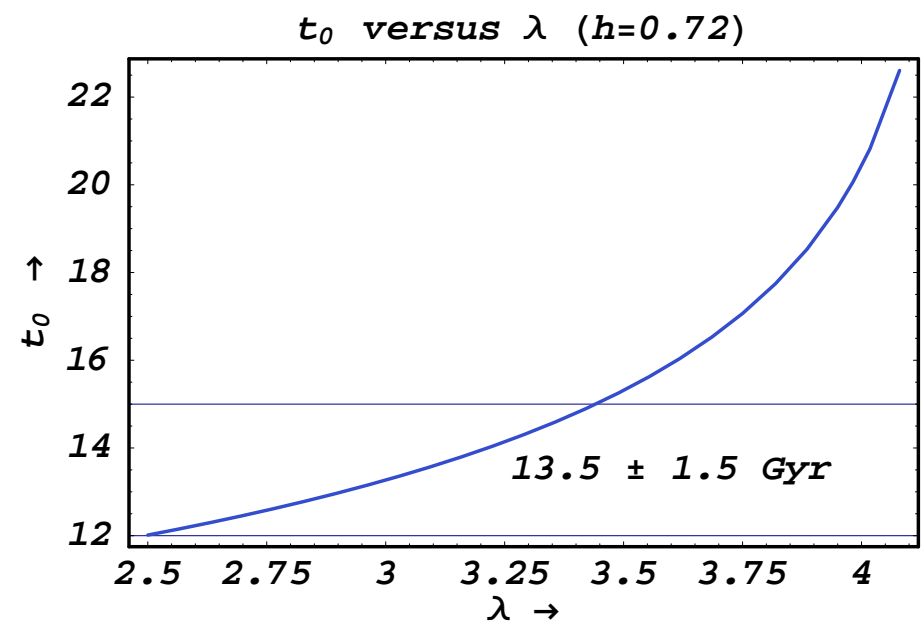

Fig. 1. The present cosmic epoch of $13.5 \pm 1.5$ Gyr corresponds to $2.50 \leq \lambda \leq 3.45$ (for $\mathrm{h}=0.72$ ). It can be shown that for $\mathrm{h}=0.64$, the range reduces to roughly $1.3 \leq \lambda \leq 2.4$, while for $h=0.80$ the present cosmic epoch of $13.5 \pm 1.5$ Gyr corresponds to roughly $3.7 \leq \lambda \leq 4.6$.

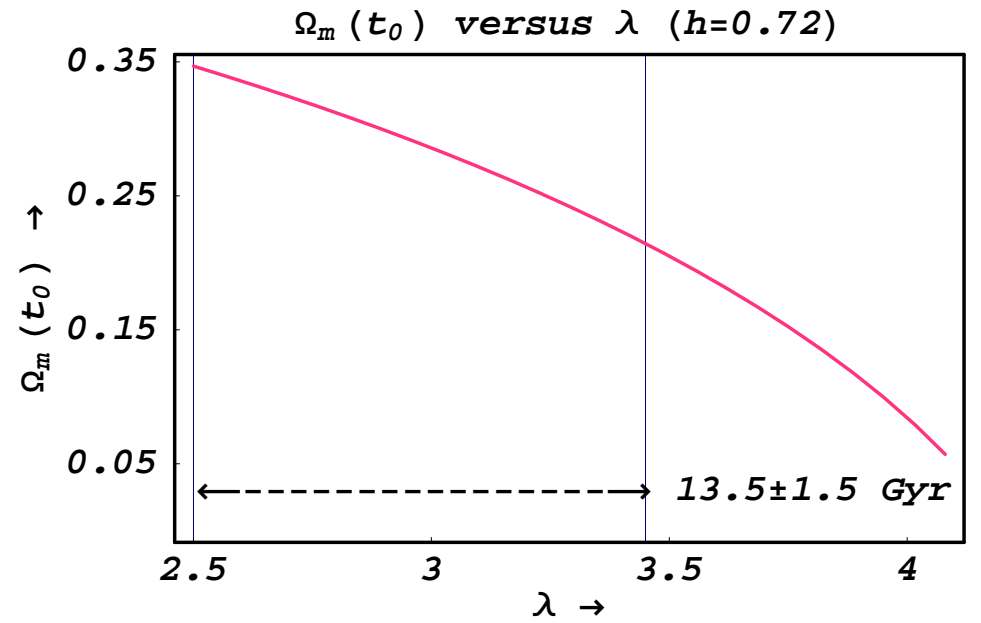

Fig. 2. The variation of $\Omega_{\mathrm{m}}$ with $\lambda$. For the present cosmic epoch $0.22 \leq \Omega_{\mathrm{m}} \leq 0.35$.

for the present epoch, the cosmic energy budget carries some three to six times as much dark matter as that contributed by the particles of the standard model of the high energy physics.

Much of the above considerations were confined to $h=0.72$. Figure 4 shows that a similar result holds for the entire observationally allowed range $0.64 \leq h \leq 0.80$. That is, the arrived dark matter existence in the present cosmic epoch is not specific to $h=0.72$.

The whole analysis can be put in the following 'back of the envelop' argument. 


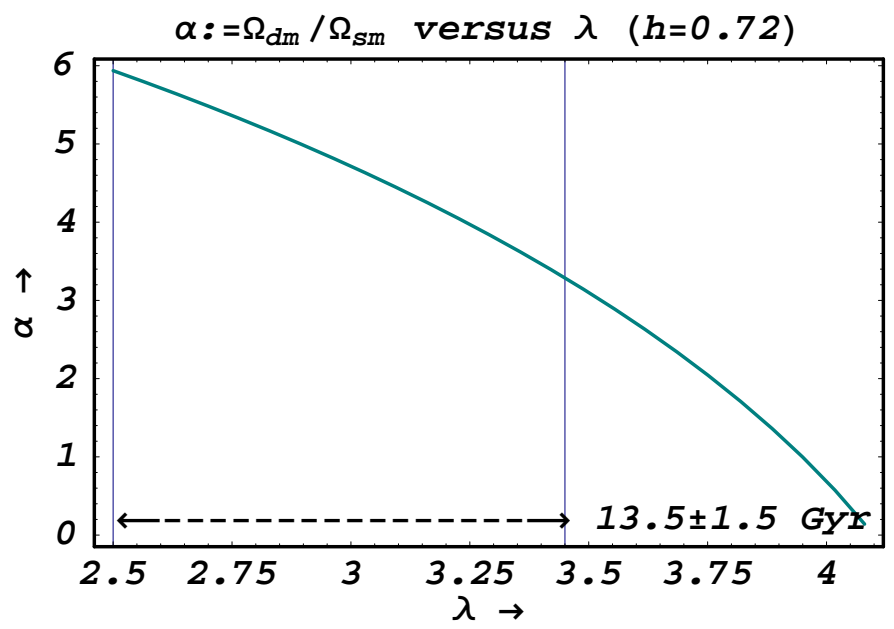

Fig. 3. The plot of $\alpha$ as a function of $\lambda$ for $\Omega_{\mathrm{sm}}=0.05$; showing that there is roughly three to six times more dark matter as compared to the total contribution from the particles of the standard model of high energy physics.

For $h=0.72$, the stellar age bound constrains the Einsteinian cosmological constant - see Eq. (10) - to $\lambda \approx 3.0$. This gives $\tau_{\Lambda} \approx 9.3 \mathrm{Gyr}$. For the present epoch of roughly 13.5 Gyr (corresponding to $\lambda \approx 3.0$ ), Eq. (7) yields $\Omega_{\mathrm{m}} \approx 0.28$. However, $\Omega_{\mathrm{sm}} \approx 0.05$ can only account for a small fraction of this obtained value. This leaves the fractional density $\Omega_{\mathrm{dm}}:=\Omega_{\mathrm{m}}-\Omega_{\mathrm{sm}} \approx 0.23$ to point towards some form of non-standard model matter in the non-relativistic form. This, by definition, is the astrophysical/cosmic dark matter [40].

The dramatic discordance between the calculated $\Omega_{\mathrm{m}}$ and the observed $\Omega_{\mathrm{sm}}$ may be alternatively interpreted, not as pointing towards the existence of some non-standard model matter (i.e., dark matter), but as a breakdown of the general relativistic description of gravitation at large astrophysical and cosmological length scales. Such a degeneracy in possible interpretations is best resolved by experimental and observational efforts. In the remainder of this essay we shall follow the darkmatter interpretation and find that it may indeed be pointing towards a very novel form of matter.

\section{Dark matter: Its darkness}

All fermionic fields of the standard model of particle physics may be expressed in terms of the Dirac spinors. Formally, these spinors live in the $(1 / 2,0) \oplus(0,1 / 2)$ representation space 43, 44. Since the 1962 work of Wigner it is known that these may not describe the entire physical reality of the high energy physics [45. Yet, till late last year, mostly due to non-trivial technical reasons and for an apparent lack of physical motivation (i.e, before the suggestions of dark matter was taken seriously), no concrete construct of the type suggested by Wigner existed for spin 
one half [46].

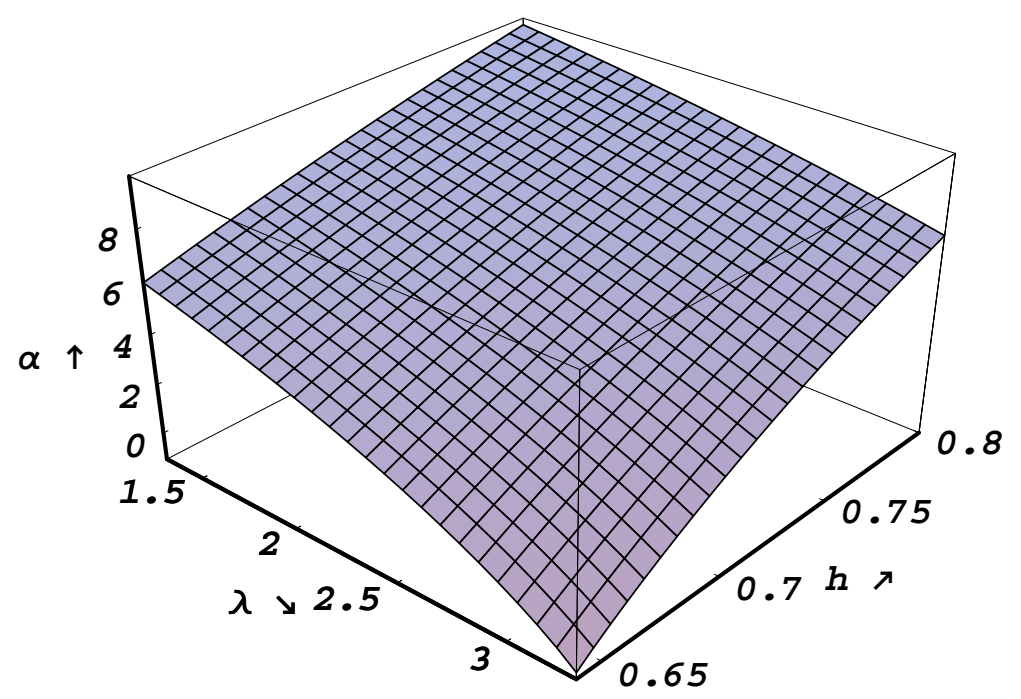

Fig. 4. The variation of $\alpha$ as a function of $\lambda$ and $h$ for $\Omega_{\mathrm{sm}}=0.05$. It shows that for almost the entire displayed range, $1.3 \leq \lambda \leq 3.3$ and $0.64 \leq h \leq 0.80$, the dark matter measuring parameter $\alpha$ remains significantly away from zero. Note parenthetically, that the denominator of Eq. [11a yields physically meaningless region just beyond $\lambda=3.3$ around $h=0.64$. This is the region at the lowest right hand corner of the above plot. And, only in this extreme region does $\alpha$ hover around zero.

To heuristically arrive at the conclusion of Wigner's notes, as he called them, we first make the obvious observation that the indicated representation space supports various symmetries. Of these, the charge conjugation $(C)$, the parity $(P)$, and time reversal $(T)$ symmetries are the most relevant for the argument. The Dirac spinors are the eigenspinors of the $P$ operator. Now $C$ and $P$ when acting upon these spinors do not commute; instead, they anti-commute. Consequently, a quantum field built upon the eigenspinors of the $C$ operator should describe physically distinct type of new particles.

A brief report on the construction of such a quantum field was recently presented in [12] while the monographic details were archived in [13]. The ensuing result states that the quantum field based on the eigenspinors of the $C$ operator does not carry the Dirac propagator but, instead, that due to Klein and Gordon. This endows the new field with mass dimension one, rather than three half as for the Dirac field. This happens despite the fact that the new field is fermionic, and carries spin one half. It is endowed with several novel features. A few of these are summarised in Table I. 
The first two properties enumerated in the second column are in accord to formal expectations based on Wigner's work [45, while da Rocha and Rodrigues have shown [49] that the new field is class 5 in Lounesto spinor classification [50. The field carries a 'minimal' element of non-locality as required by the 1966 work of Lee and Wick [51] on Wigner classes and may be important for resolving the 'horizon problem' in cosmology.

The third and the fourth results are intertwined. In contrast, they have emerged as a surprise. Unexpectedly. The mass dimensionality of the new field immediately places it out of bounds for essentially all standard model interactions. The new particles cannot be part of the standard model fermionic doublets because the mass dimensionalities mismatch. The most dominant interaction which the new particles can carry is with the Higgs [52].

In addition, and unlike the standard model fermions where the quartic self interaction is suppressed by two powers of Planck mass, the new particles carry an unsuppressed, and crucial [53, quartic self interaction. This is because of their mass dimensionality one. These aspects endow the new particles with a darkness with respect to detectors made of the terrestrial standard model material and makes them natural dark matter candidates. Due to its over abundance with respect to the standard model matter in the astrophysical and cosmological environments, the gravitational effects of dark matter remain as its primary signature. It is there that dark matter primarily manifests its presence and its consequences.

Given the role of Higgs in its interactions with the new dark matter candidate one may expect its appearance at Higgs factories. By the same token, its effects in early universe, particularly in the context of matter-antimatter asymmetry, may be profound. This is due to the mismatch of the CPT phases at the interface with the standard-model matter. But here, our emphasis is on obtaining the darkness. The new construct provides it in a natural fashion without extending the spacetime symmetries, say to super-symmetry.

Lest these results acquire a mysterious life let us hasten to add a few remarks to help evaporate any such feeling. The entire physical content of the seventy six pages of published calculations and discussions in references [12, 13] essentially resides in the fact that the $C, P$, and $T$ properties, and the emergent propagators, of the quantum fields is carried by the relative helicities and phases of the $(1 / 2,0)$ and $(0,1 / 2)$ - transforming components of the $(1 / 2,0) \oplus(0,1 / 2)$ spinors. For the eigenspinors of the $P$ (i.e., spinors underlying the Dirac field), the relative helicities of the said components are same. For the eigenspinors of the $C$ (i.e., the spinors underlying the new spin one half field), the relative helicities of the said components are opposite. The remaining degree of freedom in the relative phases between these spinorial components is fixed by requiring that these be such that they become $P$ and $C$ eigenspinors. Once this is appreciated the eigenspinors in the rest frame, apart from a normalisation factor, are uniquely determined. The $(1 / 2,0) \oplus(0,1 / 2)$ boost then furnishes us a set of complete spinors. One set of four spinors for the Dirac case, and the other for the new construct. The propagator then turns out to 
depend on the respective spin sums. For the Dirac case these spin sums have the usual $\frac{1}{m} \gamma^{\mu} p_{\mu} \pm \mathbb{1}$ form and yield the celebrated Dirac propagator. For the new field these spin sums take the form $\mathbb{1} \pm \mathcal{G}(\mathbf{p})$, and yield the propagator

$$
\mathcal{S}\left(x-x^{\prime}\right)=\int \frac{d^{4} p}{(2 \pi)^{4}} \mathrm{e}^{i p_{\mu}\left(x^{\mu}-x^{\prime \mu}\right)} \frac{\mathbb{1}+\mathcal{G}(\mathbf{p})}{p_{\mu} p^{\mu}-m^{2}+i \epsilon}
$$

The limit $\epsilon \rightarrow 0^{+}$is understood. A simple calculation using the eigenspinors of $C$ shows that $\mathcal{G}(\mathbf{p})$ is an odd function of $\mathbf{p}: \mathcal{G}(\mathbf{p})=-\mathcal{G}(-\mathbf{p})$. This has the consequence that in the absence of a preferred direction - a circumstance which may be spoiled by existence, say, of an external gravitomagnetic field - the integration over $\mathcal{G}(\mathbf{p})$ vanishes. This leaves behind a Klein-Gordon propagator multiplied by an identity matrix in the $(1 / 2,0) \oplus(0,1 / 2)$ representation space.

Table I. Some of the properties of the new field as compared to that of Dirac. Here, $\mathbb{1}$ represents a $4 \times 4$ identity matrix in the $(1 / 2,0) \oplus(0,1 / 2)$ representation space. For the commutator and anti-commutator relations it is implicit, as a knowledgeable reader knows, that their validity holds while acting on the relevant spinors. Same holds for the statement on $(C P T)^{2}$. The propagators are obtained by calculating the vacuum expectation value of the relevant time ordered product of the field operators. The calculational details appear in [12, 13.

\begin{tabular}{|l|l|}
\hline $\begin{array}{l}\text { Quantum field based on } \\
\text { Eigenspinors of } P[48]\end{array}$ & $\begin{array}{l}\text { Quantum field based on } \\
\text { Eigenspinors of } C[12,[13]\end{array}$ \\
\hline$\{P, C\}=0$ & {$[P, C]=0$} \\
$(C P T)^{2}=+\mathbb{1}$ & $(C P T)^{2}=-\mathbb{1}$ \\
Propagator: Dirac & Propagator: Klein-Gordon $\times \mathbb{1}$ \\
Mass dimension: $3 / 2$ & Mass dimension: 1 \\
\hline
\end{tabular}

Should the reader wonder as to where did the Lagrangian enter; the answer is, nowhere. And, that is the beauty of Wigner-like arguments we constructed. It is now obtained by 'inverting' the propagator. Similarly, the new construct does not apply to Majorana neutrinos. Was that to be done, they would cease to be part of the standard model doublets due to mass dimensionality carried by the new construct. A more detailed discussion on the subject can be found in the already cited references. Here it suffices to note that the 1937 Majorana proposal is not at the level of representation space [54. It still uses Dirac spinors. Only at the level of Fock space does it identify the 'particle-' and 'antiparticle-' creation and destruction operators. 


\section{Conclusion}

If one insists on the validity of the general relativistic description of gravitation on astrophysical and cosmological length scales, then the considered FriedmannRobertson-Walker cosmology with Einsteinian cosmological constant, and vanishing spatial curvature constant, unambiguously requires significant amount of dark matter. This requirement is consistent with other indications for dark matter. The same spacetime symmetries that underlie the freely falling frames of Einsteinian gravity also provide symmetries, which for the spin one half representation space, furnish a novel construct that carries extremely limited interactions with respect to the terrestrial detectors made of the matter described by the standard model of high energy physics. A case is made that both the 'luminous' and the 'dark' matter are residents of the same representation space but they derive their respective 'luminosities' and 'darkness' from being either the $(C P T)^{2}=+\mathbb{1}$ carrying eigenstates of the parity operator, or the $(C P T)^{2}=-\mathbb{1}$ carrying eigenstates of the charge conjugation operator.

\section{Acknowledgments}

I thank Daniel Grumiller for our ongoing discussions and for his permission to include some of the wisdom of our joint publications on darkness of dark matter in this essay. I thank Garth Antony Barber for our discussion on CosmoCoffee [55]. It helped me to make some of the claims more precise. It is also a pleasure to thank Thanu Padmanabhan for his constructive remarks on the first draft of this essay. To the younger blood, which remains anonymous, thank you for your fan mail. Many of these ideas crystallised, and took a new form, during our February-March vacation in Australia. For that I thank Karan Ahluwalia, his family, and my wife, for creating that wonderful space and time where one can think in an inspired manner.

Much of this work was done at the Department of Mathematics, University of Zacatecas (Mexico). Extended hospitality of that institution, and in particular that of its Director Gema Mercado, is greatfully acknowledged.

\section{References}

1. M. Persic, P. Salucci and F. Stel, The Universal rotation curve of spiral galaxies: 1. The dark matter connection, Mon. Not. Roy. Astron. Soc. 281, 27 (1996) arXiv:astro-ph/9506004.

2. D. M. Wittman, J. A. Tyson, D. Kirkman, I. Dell'Antonio and G. Bernstein, Detection of weak gravitational lensing distortions of distant galaxies by cosmic dark matter at large scales, Nature 405, 143 (2000) arXiv:astro-ph/0003014.

3. H. Hoekstra, H. K. C. Yee and M. D. Gladders, Properties of galaxy dark matter halos from weak lensing, Astrophys. J. 606, 67 (2004) arXiv:astro-ph/0306515.

4. M. Milgrom, A modification of the Newtonian dynamics: Implications for galaxies, Astrophys. J. 270, 371 (1983).

5. R. H. Sanders and S. S. McGaugh, Modified Newtonian dynamics as an alternative to dark matter, Ann. Rev. Astron. Astrophys. 40, 263 (2002) astro-ph/0204521. 
6. J. D. Bekenstein, Relativistic gravitation theory for the MOND paradigm, Phys. Rev. D70, 083509 (2004) astro-ph/0403694. J. D. Bekenstein, Phys. Rev. D. 71, 069901 (2005) (erratum).

7. H. S. Zhao and B. Famaey, Refining MOND interpolating function and TeVeS Lagrangian, Astrophys. J. 638 (2006) L9 arXiv:astro-ph/0512425].

8. Y. Sobouti, An $f(R)$ gravitation instead of dark matter, arXiv:astro-ph/0603302

9. D. V. Ahluwalia-Khalilova, Dark matter: A phenomenological existence proof, arXiv:astro-ph/0601489

10. R. G. Vishwakarma, "Comments on Dark matter: A phenomenological existence proof," arXiv:astro-ph/0603213.

11. D. V. Ahluwalia-Khalilova, "A reply to a comment on 'Dark matter: A phenomenological existence proof'," arXiv:astro-ph/0603256

12. D. V. Ahluwalia-Khalilova and D. Grumiller, Dark matter: A spin one half fermion field with mass dimension one?, Phys. Rev. D $\mathbf{7 2}$ (2005) 067701 arXiv:hep-th/0410192.

13. D. V. Ahluwalia-Khalilova and D. Grumiller, Spin half fermions with mass dimension one: Theory, phenomenology, and dark matter, JCAP 0507 (2005) 012 arXiv:hep-th/0412080.

14. S. Perlmutter et al. [Supernova Cosmology Project Collaboration], Discovery of a supernova explosion at half the age of the universe and its cosmological implications, Nature 391 (1998) 51 arXiv:astro-ph/9712212.

15. A. G. Riess et al. [Supernova Search Team Collaboration], Observational evidence from supernovae for an accelerating universe and a cosmological Constant, Astron. J. 116, 1009 (1998) arXiv:astro-ph/9805201.

16. S. Perlmutter et al. [Supernova Cosmology Project Collaboration], Measurements of $\Omega$ and $\Lambda$ from 42 high-redshift supernovae, Astrophys. J. 517, 565 (1999) arXiv:astro-ph/9812133.

17. J. L. Tonry et al. [Supernova Search Team Collaboration], Cosmological results from high-z supernovae, Astrophys. J. 594 (2003) 1 arXiv:astro-ph/0305008.

18. A. G. Riess et al. [Supernova Search Team Collaboration], Type Ia Supernova discoveries at $z>1$ from the Hubble Space Telescope: Evidence for past deceleration and constraints on dark energy evolution, Astrophys. J. 607, 665 (2004) arXiv:astro-ph/0402512.

19. P. Astier et al., The Supernova Legacy Survey: Measurement of $\Omega_{m}, \Omega_{\Lambda}$ and $w$ from the first year data set, arXiv:astro-ph/0510447.

20. H. K. Jassal, J. S. Bagla and T. Padmanabhan, Observational constraints on low redshift evolution of dark energy: How consistent are different observations?, Phys. Rev. D 72 (2005) 103503 arXiv:astro-ph/0506748.

21. H. K. Jassal, J. S. Bagla and T. Padmanabhan, The vanishing phantom menace, arXiv:astro-ph/0601389

22. D. N. Spergel et al. [WMAP Collaboration], First year Wilkinson Microwave Anisotropy Probe (WMAP) observations: Determination of cosmological parameters, Astrophys. J. Suppl. 148 (2003) 175 arXiv:astro-ph/0302209.

23. E. J. Copeland, M. Sami and S. Tsujikawa, Dynamics of dark energy, arXiv:hep-th/0603057

24. D. L. Wiltshire, Viable inhomogeneous model universe without dark energy from primordial inflation, arXiv:gr-qc/0503099

25. H. Alnes and M. Amarzguioui, The supernova Hubble diagram for off-center observers in a spherically symmetric inhomogeneous universe," arXiv:astro-ph/0610331

26. N. Mustapha, B. A. Bassett, C. Hellaby and G. F. R. Ellis, "Shrinking II - The Distor- 
tion of the Area Distance-Redshift Relation in Inhomogeneous Isotropic Universes," Class. Quant. Grav. 15 (1998) 2363 arXiv:gr-qc/9708043.

27. S. Rasanen, "Accelerated expansion from structure formation," JCAP 0611 (2006) 003 arXiv:astro-ph/0607626.

28. B. M. N. Carter, B. M. Leith, S. C. C. Ng, A. B. Nielsen and D. L. Wiltshire, Exact model universe fits type IA supernovae data with no cosmic aacceleration, arXiv:astro-ph/0504192

29. For additional discussion, see, e.g., 30, 31

30. H. Alnes, M. Amarzguioui and O. Gron, An inhomogeneous alternative to dark energy?, arXiv:astro-ph/0512006

31. T. Buchert, On globally static and stationary cosmologies with or without a cosmological constant and the dark energy problem, Class. Quant. Grav. 23 (2006) 817 arXiv:gr-qc/0509124.

32. O. Gron, A new standard model of the universe, Eur. J. Phys. 23 (2002) 135-144.

33. J. A. Frieman, The standard cosmology, arXiv:astro-ph/9404040.

34. K. Lake, Integration of the Friedmann equation for universes of arbitrary complexity, arXiv:gr-qc/0603028.

35. If $w^{\Lambda}=p^{\Lambda} / \rho^{\Lambda}$ is confined to the choice $w^{\Lambda}=-1$ then a time-independent $\rho^{\Lambda}$ corresponds to the Einsteinian cosmological constant.

36. This result is under the assumption that one may discard negligible contribution coming from radiation and from the radiation-dominated epoch.

37. E. W. Kolb and M S. Turner, The early universe (Westview Press, 1990)

38. W. L. Freedman et al., Final results from the Hubble Space Telescope Key Project to measure the Hubble constant, Astrophys. J. 553 (2001) 47 arXiv:astro-ph/0012376.

39. B. Chaboyer, Astrophysical ages and time scales, in: T. von Hippel, C. Simpson, N. Manset (Eds.), APS Conference Series, Vol. 245, 2001, p.162.

40. In a recent preprint Balasin and Grumiller have shown 41 that Newtonian arguments over-estimate the amount of matter needed to explain non-Keplerian galactic rotation curves by more than thirty percent. This decrease in demand on dark matter content would need to be accounted for in more careful estimation of dark matter from the galactic rotation curves and gravitational lensing data. For care that needs to be exercised in such joint analysis, see Faber-Visser preprint 42

41. H. Balasin and D. Grumiller, Significant reduction of galactic dark matter by general relativity, arXiv:astro-ph/0602519.

42. T. Faber and M. Visser, Combining rotation curves and gravitational lensing: How to measure the equation of state of dark matter in the galactic fluid, arXiv:astro-ph/0512213

43. S. Weinberg, The quantum theory of fields, Vol. I (Cambridge University Press, Cambridge, 1995)

44. L. H. Ryder, Quantum field theory (Cambridge University Press, Cambridge, 1997).

45. E. P. Wigner, in Group theoretical concepts and methods in elementary particles physics: Lectures of the Istanbul summer school of theoretical physics (July 16-August 4, 1962), pp. 37-80, Editor F. Gürsey (Gordon and Breach, New York, 1964).

46. For work on spin one, see Ref. 47.

47. D. V. Ahluwalia, M. B. Johnson and T. Goldman, A Bargmann-Wightman-Wigner type quantum field theory, Phys. Lett. B 316 (1993) 102 arXiv:hep-ph/9304243.

48. P. A. M. Dirac, The quantum theory of electron, Proc. Roy. Soc. Lond. A 117 (1928) 610.

49. R. da Rocha and W. A. J. Rodrigues, Where are ELKO spinor fields in Lounesto spinor field classification?' Mod. Phys. Lett. A 21 (2006) 65 arXiv:math-ph/0506075. 
50. P. Lounesto, Clifford algebras and spinors (Cambridge University Press, Cambridge, 1997).

51. T. D. Lee and G. C. Wick, Space inversion, time reversal, and other discrete symmetries in local field theories, Phys. Rev. 148 (1966) 1385.

52. See, references 12,13 for additional discussion.

53. K. J. Ahn and P. R. Shapiro, Formation and evolution of the self-interacting dark matter halos, Mon. Not. Roy. Astron. Soc. 363 (2005) 1092 arXiv:astro-ph/0412169.

54. E. Majorana, Theory of the symmetry of electrons and positrons, Nuovo Cim. 14 (1937) 171. An English translation of this 1937 classic Italian language paper by L. Maiani can be found in Soryushiron Kenkyu 63 (1981) 149. The reader should feel free to request a pdf file from the author of this essay.

55. G. Barber and D. V. Ahluwalia-Khalilova, http://cosmocoffee.info/viewtopic.php?p=1409\#1409 